\title{
Improved Denoising Method for Ultrasonic Echo with Mother Wavelet Optimization and Best-Basis Selection
}

\author{
Mohammad Hossein Doost Mohammadi \\ Department of Electrical Engineering, Hamedan University of Technology, Iran
}

\begin{tabular}{l}
\hline \hline Article Info \\
\hline Article history: \\
Received Mar , 2016 \\
Revised Jun 22, 2016 \\
Accepted Jul 5, 2016 \\
\hline
\end{tabular}

Keyword:

Denoising

Discrete wavelet transform

Threshold

Ultrasonic_NDT

\begin{abstract}
Weak features of ultrasonicnondestructive test signals are usually immersed in noisy signals. So, in this paper, we proposed an improved scheme for noise reduction and feature extraction based on discrete wavelet transform. The basis of the mother wavelet was selected to be matched to a given signal. Three different constraints were presented to minimize the error between the denoised and the given signal. It should be mentioned that such an optimum wavelet can represent the signal more compactly with a few large coefficients which can be considered as the signal features. Standard signals and simulated ultrasonic echo were used to evaluate the performance of the presented algorithms. Signal to error ratio was used to compare the designed wavelet performance with that of standard wavelets. Simulation results revealed that the proposed method outperformed the other presented methods and even standard wavelets. The results also has shown that the signal-based noise reduction algorithms make the feature extraction more reliable. Finally, the performance of the proposed algorithm was compared with other methods from different literatures.
\end{abstract}

Copyright (c) 2016 Institute of Advanced Engineering and Science. All rights reserved.

\section{Corresponding Author:}

Mohammad Hossein Doost Mohammadi, Department of Electrical Engineering, Hamedan University of Technology, Mardom St., Shahid Fahmideh Blv. Hamedan, Iran.

Email: doost.mohammadi@hut.ac.ir

\section{INTRODUCTION}

Ultrasonic_NDT is a measure utilized in many industries for identifying special features and defects in the process of producing various parts and materials, in order to warranty the required quality and reliability of the finished products. Ultrasonic_NDT could also be used in analyzing body tissues and detecting diseases in medical practices. It goes without saying that accuracy and precision are of salient importance in both these fields. However, due to environmental factors and the subject of the test itself, these signals usually include noises that are in the same frequency range as the reflected signals from the defections. This could result in an inability to identify the weak features and defects that would decrease the reliability and accuracy of this test. Therefore, these signals require a pre-processing step for noise reduction.

So far, several signal processing methods in time and frequency domains have been proposed. Mohammed et al. presented a new idea of noise reduction based on two stage: adaptive line enhancer (ALE) and normalized least mean square (NLMS) [1]. He also proposed an adaptive noise canceller for remote health monitoring which was a combination of adaptive notch filter and modified LMS algorithm [2]. More classic algorithms can be found in the survey literatures [3].

One of the successful methods in noise reduction is using wavelet transform (WT). It has been illustrated that WT yields a more precise result in identifying the signals from noise in comparison to other methods, such as common used filtering or Wiener filtering methods. One of the main underlying reasons is the concentration of signal energy on a limited number of coefficients. Several parameters should be 
considered in using this method, parameters such as the wavelet filters and the threshold [4]. Most of these wavelet based methods use standard wavelets or a combination of standard wavelets and classic filtering methods [5]. Even though this method could be an efficient way for reducing noises, but it is not designed for a specific signal and is not completely adjusted for each subject of experiment.

The wavelet decomposition is determined by mother wavelet function and its dilation and shift versions [4]. Since, many works have been presented by researchers to find wavelets matched to signals providing the best representation for a given signal. Daubechies presented methods to find orthonormal and biorthonormal wavelet bases with compact support $[6,7]$. The resulting wavelets were acceptable according to constraints but both of these wavelet design methods were independent of the signals being analyzed. Gupta et al. presented a method in the time domain based on maximizing the projection of the given signal into a successive scaling subspace and minimization in the wavelet subspace [8-10]. Tewfik et al. presented a method which led to finding the best estimation of the desired signal $f(t)$ with integer translates of a valid scaling function of finite fixed support $N$, dilated by a given factor $M$, at the proper scale $J$ [11]. Actually in [11] the upper bound of error norm has been minimized instead of minimizing the actual $L^{2}$ distance between $f(t)$ and $f^{J}(t)$. Gopinath et al., has done the minimization of norm in the frequency domain instead of time domain due to its complexity [12]. The minimization of frequency domain $L^{P}$ norm of the approximation error was used to reach the optimality. But the major problem was the complexity of equations that are very difficult to solve.

Mallat and Zhang proposed an algorithm that decomposes any signal into a linear expansion of waveforms that are selected from a redundant dictionary of functions [13]. Similar work has been presented by Krim based on minimization of reconstruction error of the underlying signal [14]. In [14] selecting an optimal basis is done among a family of known basis instead of constructing a wavelet matched to the statistics of the given signal.

Rao and Chapa have presented a method to design a wavelet that looks like a given signal for the case of orthonormal multiresolution analysis with band limited wavelets [15]. But that method was computationally expensive. Similar work has been done by $\mathrm{Wu}$-sheng and Tsatsanis in which the algorithms led to very complicated solutions [16-17].

In this paper, we present three methods for designing optimized wavelet which are based on minimization of nonlinear estimation error. Presented methods have no major complexity and could be implemented simply. These methods could yield a considerable improvement in SNR and feature identification. Next, we will perform our optimized noise reduction methods in simulated ultra-sound echoes and analyze the resulting level of optimization in identifying their weak features and related SNR.

\section{BACKGROUND AND THEORY}

\subsection{Discrete Wavelet Transform}

As described in the wavelet literature [18], by using the discrete wavelet transform (DWT), a function $y(x) \in L^{2}(R)$ can be expanded as:

$$
y(t)=\sum_{k} c_{j_{0} k} \phi_{j 0, k}(x)+\sum_{k} \sum_{j=j_{0}}^{\infty} d_{j k} \psi_{j, k}(x)
$$

where $\phi_{j, k}(x)=2^{j / 2} \phi\left(2^{j} x-k\right)$ is called the scaling function and $\psi_{j, k}(x)=2^{j / 2} \psi\left(2^{j} x-k\right)$ is called the wavelet function. $j$ and $k$ are called dilation and translation parameters respectively and $j_{0}$ is the coarsest scale in the decomposition. $d_{j k}$ and $c_{j k}$ are the detail and approximation coefficients and they can be calculated by inner products for orthogonal wavelet system as follow [1], [14]:

$$
\begin{aligned}
& d_{j k}=\left\langle y(x), \psi_{j, k}(x)\right\rangle \\
& c_{j k}=\left\langle y(x), \phi_{j, k}(x)\right\rangle
\end{aligned}
$$

Using the basic recursion, $\phi(x)$ and $\psi(x)$ can be written as [14]:

$$
\phi(x)=\sqrt{2} \sum_{k=-\infty}^{\infty} h[k] \phi(2 x-k)
$$


$\psi(x)=\sqrt{2} \sum_{k=-\infty}^{\infty} g[k] \phi(2 x-k)$

where $g$ and $h$ are impulse response of quadrature mirror filters. These filters are used to implement the fast wavelet transform and should satisfy the conditions of orthogonality $[18,19]$, as follow:

$$
\begin{aligned}
& \sum_{k} h[k]=\sqrt{2} \\
& \sum_{k} h[k] h[k-2 n]=\delta[n] \\
& \sum_{k}(-1)^{k} k^{m} h[k]=0 \text { for } m=0, \ldots, M-1
\end{aligned}
$$

Decomposition is performed using these filters. It's been shown that $\psi_{j, n}$ and $\phi_{j, n}$ will construct an orthogonal basis for $L^{2}(R)$, if $g$ is selected as:

$$
g[k]=(-1)^{1-k} h[1-k]
$$

or

$$
G(\omega)=e^{-j \omega} H^{*}(\omega+\pi)
$$

where $H$ and $G$ are the Fourier transform of $h$ and $g$, respectively.

\subsection{Wavelet Estimation}

Our goal is data estimation from noisy signals. Let's consider $Y[n]$ as the noisy signal, $f[n]$ as the signal to be estimated and $\omega[n]$ as the Gaussian noise (white or colored). So, it can be shown that [4]:

$$
Y[n]=f[n]+\omega[n]
$$

For signal estimation using wavelets, $N$ samples of the noisy signal $Y[n]$ are decomposed in the discrete wavelet basis which is defined over $[0, N-1][4]$ :

$$
\left[\left\{\psi_{j, m}\right\}_{j_{0} \leq j<\infty, 0 \leq m<N 2^{j}},\left\{\phi_{j 0, m}\right\}_{0 \leq m<N 2^{j_{0}}}\right]
$$

In this basis, the estimate of $f[n]$ is written as

$$
\tilde{f}=\sum_{j=j_{0}}^{\infty} \sum_{m=0}^{N 2^{j}} \theta_{h}\left(\left\langle\psi_{j, m}, Y\right\rangle\right) \psi_{j, m}+\sum_{m=0}^{N 2^{j_{0}}} \theta_{h}\left(\left\langle\phi_{j_{0}, m}, Y\right\rangle\right) \phi_{j_{0}, m}
$$

where $\theta_{h}(x)$ is called the threshold function. By minimizing the mean square error, the best estimate of $f[n]$ sould be achieved [4]:

$$
\epsilon=\mathrm{E}\{\|f-\tilde{f}\|\}^{2}
$$

Using a hard threshold for denoising, can lead tosmallererror if a few decomposition coefficients of the noisy signal are above the threshold. So, it can be said that a good approximation of $f$ has been achieved by them, as can be implied from [4]. Thus we may loosely say that our optimum wavelet base should provide the best nonlinear estimation of the signal $f$. In other words, the error of signal estimation with $M$ largest wavelet expansion coefficients should be minimum:

$$
\epsilon[m]=\left\|f-f_{M}\right\|^{2}=\sum_{k=M+1}^{+\infty}\left|\left\langle f, g_{m_{k}}\right\rangle\right|^{2}
$$


According to the notation of [4], $f_{M}$ is the signal reconstructed with $M$ largest wavelet coefficients and $g_{m_{k}}$ are the basis vectors on which the signal projections are larger. It can be said that the error itself is related to the decay speed of the expansion coefficients. So, the relation could be qualified by the $l^{p}$ norm of the expansion coefficients of the signal in the basis [4], [20]:

$$
\|f\|_{B, p}=\left(\sum_{m=0}^{+\infty}\left|\left\langle f, g_{m}\right\rangle\right|^{p}\right)^{\frac{1}{p}}
$$

where $B$ is the basis on which the signal is begin expanded. According to the notation of [4], if $\|f\|_{B, p}<\infty$ and $p<2$, then we have:

$$
\epsilon[m] \leq \frac{\|f\|_{B, p}^{2}}{\frac{2}{p}-1} M^{1-\frac{2}{p}}
$$

\subsection{Theresholding Methods} include [21]:

As shown in Figure 1, there are various methods of basic thresholding approaches, which

a. Hard thresholding, where all the coefficients below a predefined threshold value are set to be zero.

b. Soft thresholding, where in addition the remaining value are linearly reduced in value.

c. Nonlinear thresholding, the original coefficients are mapped to a new set, using a smooth function to avoid abrupt value changes.

Thresholds can be estimated from the wavelet coefficients $x_{i j}$ of each scale $i$. In the following, $N_{i}$ represents the number of coefficients of scale $i$, and $\hat{\sigma}_{i}$ the standard deviation of these wavelet coefficients. Threshold selectionruleusedin this work is the MultiMAD threshold which is given by $T=\sigma \sqrt{2 \log (n)},[22-24]$.

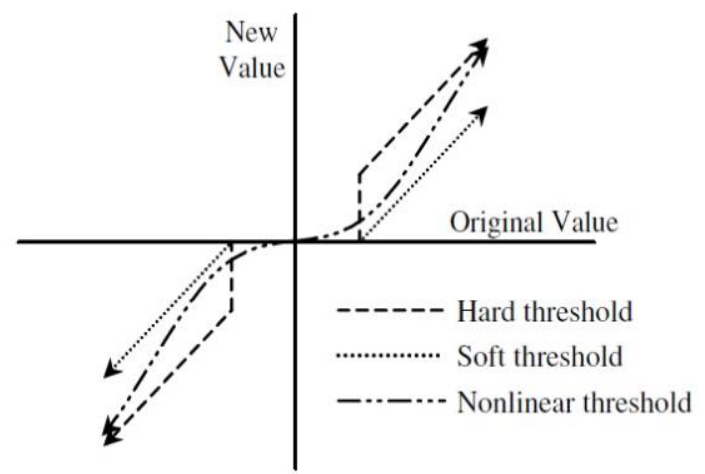

Figure 1. Threshold Mapping Functions [21]

\section{METHODOLOGY}

\subsection{Criteria for Wavelet Design}

In these section, three criteria for designing wavelets will be introduced. As explained in section 2.2, the denoising error can be reduced by reducing $\|f\|_{B, p}^{2}$. So, the main goal is finding a wavelet basis that minimizes this norm:

$$
\lambda=\min _{B}\|f\|_{B, p}^{2}
$$

where $\lambda$ is our desired cost function. Two criteria based on this cost function can be presented as follow [25].

Criteria 1\&2: A guiding principle proposed there was to aim for maximization of the variance, either maximization of the variance of the absolute values of the wavelet coefficients, or maximization of the 
variance of the squared wavelet coefficients i.e. of the energy distribution over the wavelet contributions at the various scales [25]. The theorem presented in [25] is as follow:

Let $\left\{w_{k} \mid k=0,1, \ldots, m\right\}$ be the sequence of the wavelet coefficients at all the levels and the approximation coefficients at the coarsest level resulting from the processing of a signal $x=\left(x_{0}, x_{1}, x_{2}, \ldots, x_{m}\right)$ by means of an orthogonal filter bank. Then [25]:

(a) Maximization of the variance of the sequence of absolute values $\left|w_{k}\right|$ is equivalent to minimization of the 'L1-norm' $V_{1}=\sum_{k=0}^{m}\left|w_{k}\right|$.

(b) Maximization of the variance of the sequence of energies $\left|w_{k}\right|^{2}$ is equivalent to maximization of the 'L4norm' $V_{4}=\left(\sum_{k=0}^{m}\left|w_{k}\right|^{4}\right)^{1 / 4}$

The proof of this theorem can be found in [25].

For designing a wavelet, first a specific test signal (pure and not noisy one) is chosen. Then, some random parameters are initially set as wavelet parameters and in continue, the wavelet parameters are changed to give the most optimal L1-norm and L4-norm representation of the test signal. Choosing parameters of the optimum wavelet has been done using optimization tools such as simulated annealing, neural network, and genetic algorithm. In this work, we use genetic algorithm to find parameters of the optimum wavelet. So, two wavelets have been designed using $L=6$ : one by minimizing the L1-norm of the wavelet transform of the reference signal (which will be call "Norm1" in this paper), and another one by maximizing its L4- norm (which will be call "Norm4" in this paper).

Criteria 3: Here we proposed a new method to design an optimum wavelet. In this method we set some random parameters as wavelet parameters $\left(\theta_{s}\right)$, and this wavelet is applied to a reference signal. The coefficients of the test signal will be under thresholding method and the remained coefficients will be used to reconstruct the signal. In the next step, reconstructed signal is compared to the original signal and the error signal will be extracted. In the next step, we change the wavelet parameters considering that the error signal should be minimized. Here we used genetic algorithm to find the optimum wavelet parameters that minimize the error signal. This method is called "SER".

\subsection{Genetic Algorithm}

In this work, we use genetic algorithms for selecting the optimized coefficients. Some steps should be considered, when using genetic algorithm.

a. Initial seed group: In this work we choose the seed group of size $\mathrm{P}=50$.

b. Seed selection: In this work, we choose the seed selection equal to 25 .

c. Crossover and Mutation: In this work, we used the crossover and mutation as [26].

d. Stop condition. Other parameters to be considered may be found in related literature [26].

\section{SIMULATION RESULTS AND DICUSSION}

In this section, standard test data and simulated ultrasound signal are used to evaluate the performance of the presented algorithms. First, we present the result of performing the algorithm on standard test data, and then, simulated ultrasound signal is used to compare the algorithms presented previously: "SER", "Norm1", "Norm4" and "DB3". To make it a better comparison, we have used standard wavelet Daubechies 3 (DB3) which showed the best denoisingperformance among all the other standard wavelet families. In this work, signal to error ratio (SER) is used to compare the performance of different algorithms. All simulations are performed in Matlab ${ }^{\circledR}$ software using the Wavelab toolbox [27].

\subsection{Standard Signals}

Here, we have used standard signals such as Doppler, Blocks, Heavisine and Bumps for designing the wavelets and analyzing their performance. These signals have 1024 points as shown in Figure 2 . They were first presented by Donoho and Johnstone for research in wavelet-based denoising methods [23-24]. These signals have special frequency features and have many similarities with environmental signal. To have comparable results, all the signals are normalized before simulations in order to have the same power. The simulation is performed by steps as follow.

We have used a fixed length, $L=6$, for the wavelet filters, to be able to compare different algorithms. Next, white Gaussian noise is added to the signals, and then noise reductionprocedure is performed on the corrupted signals using standard wavelet (here Daubecies3) and the wavelets designed based on criteria from the previous section. This process is performed 500 times on noises with different power levels. The mean result is considered as the final result. 


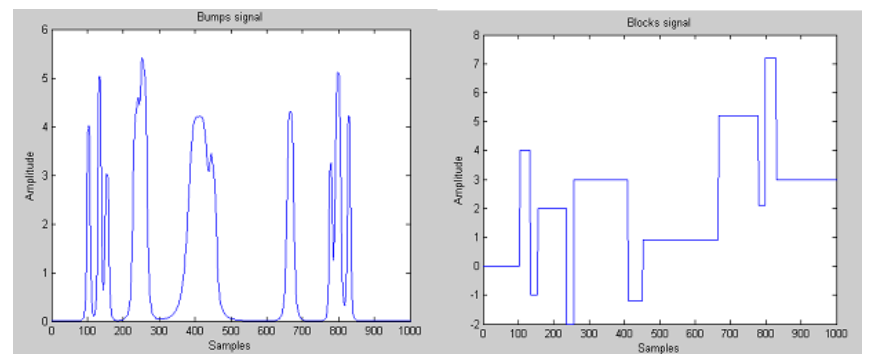

(b)

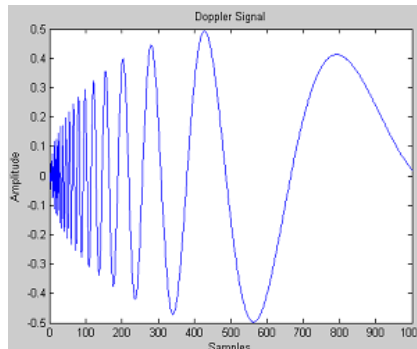

(d) (a)

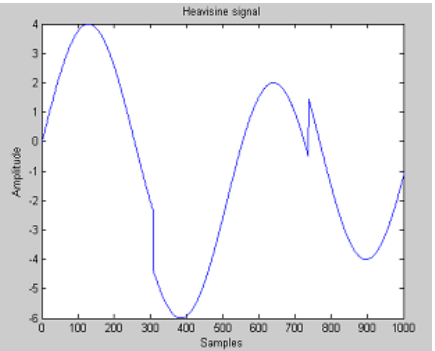

(c)

Figure 2. Standard Signals Used for Designing the Wavelets and Analyzing their Performance (a) Blocks, (b) Bumps, (c) Heavisine, (d) Doppler [6]

Table 1. Free Parameters and the Value of the Cost Function for Various Signals. The Filter Length is $L=6$

\begin{tabular}{|c|c|c|c|c|c|c|}
\hline Signal type & Algorithms & $\Theta_{1}$ & $\Theta_{2}$ & $\lambda_{\text {mine }}$ & $\lambda_{d b 3}$ & scale \\
\hline \multicolumn{7}{|l|}{ Blocks } \\
\hline & Norm1_algo & 1.8672 & -2.8430 & 7.3818 & 9.9209 & 8,9 \\
\hline & Norm4_algo & 0.43952 & 2.89224 & 1.0215 & 1.5123 & 8,9 \\
\hline & SER_algo & 0.1734 & -1.58121 & \multicolumn{3}{|c|}{$\sigma_{\text {forwaveletdesign }}=0.3$} \\
\hline \multicolumn{7}{|l|}{ Bumps } \\
\hline & Norm1_algo & 0.4041 & 3.0111 & 5.55 & 5.7231 & 8,9 \\
\hline & Norm4_algo & 3.4917 & 3.0039 & 0.3776 & 0.4831 & 8,9 \\
\hline & SER_algo & 1.29497 & -1.5026 & \multicolumn{3}{|c|}{$\sigma_{\text {forwaveletdesign }}=0.3$} \\
\hline \multicolumn{7}{|l|}{ Heavisine } \\
\hline & Norm1_algo & -2.3029 & 1.8404 & 3.9783 & 4.3424 & $6,7,8,9$ \\
\hline & Norm4_algo & -1.7444 & 1.4956 & 0.4885 & 0.8893 & $6,7,8,9$ \\
\hline & SER_algo & 0.4025 & 3.0143 & \multicolumn{3}{|c|}{$\sigma_{\text {forwaveletdesign }}=0.3$} \\
\hline \multicolumn{7}{|l|}{ Doppler } \\
\hline & Norm1_algo & 3.57975 & 2.98211 & 36.7341 & 42.1375 & $6,7,8,9$ \\
\hline & Norm4_algo & 0.80365 & 1.7920 & 3.1614 & 4.2315 & $6,7,8,9$ \\
\hline & SER_algo & 3.5649 & 2.9768 & \multicolumn{3}{|c|}{$\sigma_{\text {forwaveletdesign }}=0.3$} \\
\hline
\end{tabular}

In order to get to the required signal, we have minimized the constriction from previous section considering that the designed wavelet and scaling functions should meet the conditions given in section 2.1. Thus, the parameterization method for the decomposition filters [19] ( $h$ and $g$ ) have been used. The advantage of using this method is that the design of wavelet filter with length $2 L-1$ leads to selecting $L-1$ parameters for filter $h$ and the required conditions of section 2.1 will surely be met. Then, using equation (9), the filter $g$ can be designed. So, the cost function can be rewritten as (19):

$$
\lambda=\min _{\left\{\theta_{i}\right\}}\|f\|_{B, p}^{2}
$$


where $\theta_{i}$ are the parameters to be selected. This cost function should be minimize for the given signals in those scales used for denoising operation. It should be mentioned that this algorithm can be used not only for denoising propose, but also for providing better feature extraction. Due to providing the improved approximation of the signal, this method can well extract features of the desired signal by providing a few large wavelet coefficients as the signal's features. This features are very important because of their roles in fault diagnosis, detection and compression proposes. More details about parameterization can be found in [19]. Since we have used the fixed length filter $(\mathrm{L}=6)$, we have only two free parameter $\left(\theta_{1}\right.$ and $\left.\theta_{2}\right)$. These two parameters, along with the relatedcost function $(\lambda)$ is illustrated in the Table 1 . Result from applying different algorithms on standard signals are shown in Figures 3-6.

Examining Figure 3, the "SER" estimated signal has the better shape than others. For this 'Block' signal, the "Norm1" method generally performs well in the sharp corners but not in the flat regions (Figure 3(d)). As shown in Figure 3(c), for "SER" method, the estimated signal keep the flat region smoother than others and the corners sharp enough (but not as well as "Norm1"). So, the "Norm1" does a better job than other methods at the corners, and the "SER" method does a better job at the flat regions.

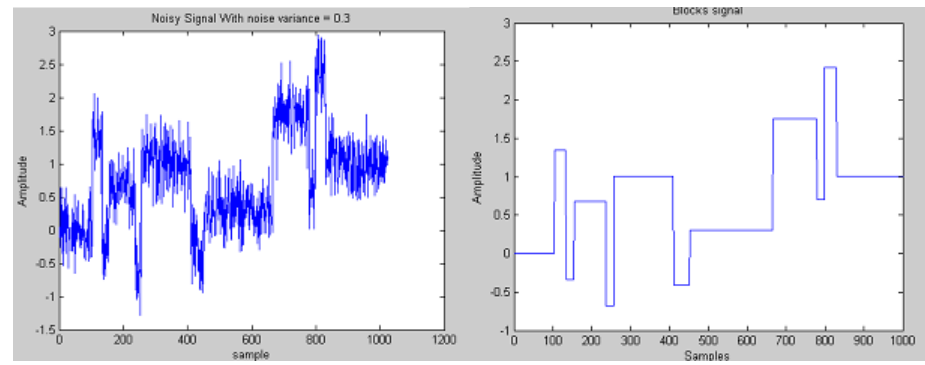

(b)

(a)

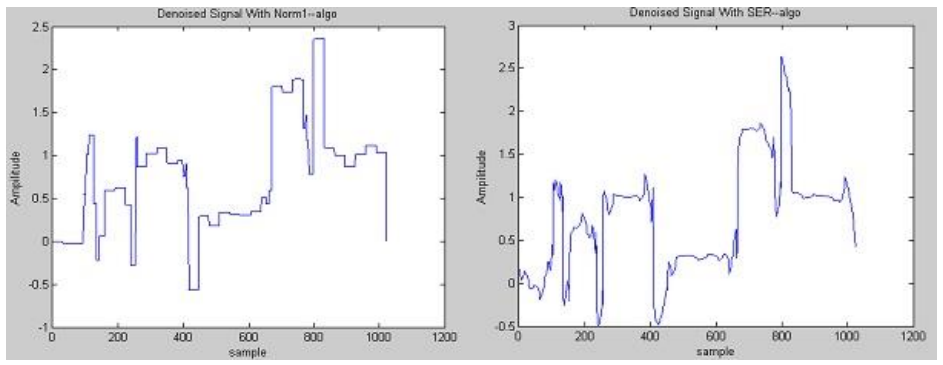

(d)

(c)

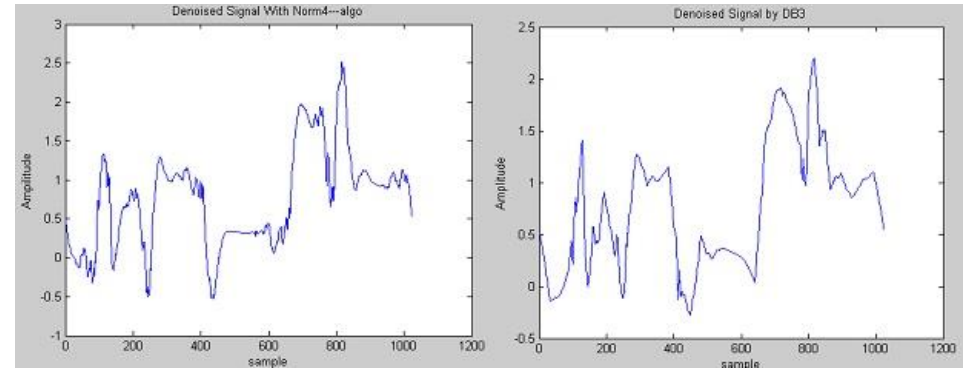

(f)

(e)

Figure 3. Results of Applying Different Wavelets to Blocks signal: (a) Pure Blocks Signal, (b) Noisy Signal with Noise Variance $\sigma=0.3$, (c) Denoised Signal by SER Algorithm, (d) Denoised Signal by Norm1 Algorithm, (e) Denoised Signal by DB3standard Wavelets, (f) Denoised Signal by Norm4 Algorithm

In Figure 4, for the 'Bumps' signal, the Norm1 estimated signal appear to be in better graphical shape. It underestimates all the cups in Figure 4(d) when compared to the original signal in Figure 4(a). The 
"SER" estimated signal is also acceptable and cleaner. As shown in Figure 4, the original signal amplitude is estimated better in "SER" method rather than "Norm1". The "Norm4" and "DB3" methods were unable to extract all the cups (Figures 4(f)-4(e)). A trend emerges from evaluating these two standard signals: (1) "SER" method seems to perform better in smooth regions and also has an acceptable jobs at sharp features. (2) "Norm1" method may mistake noise for true signal in flat and smooth regions, but the sharp features are retained.

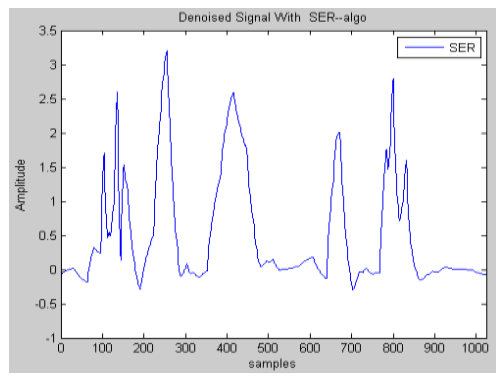

(c)

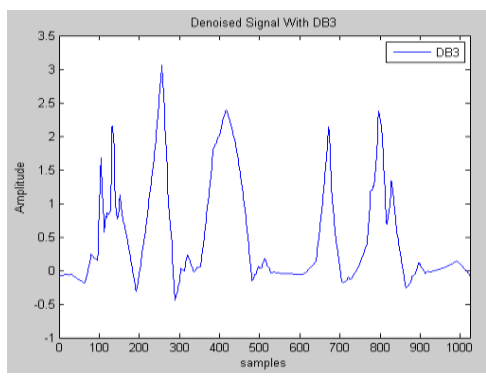

(f)

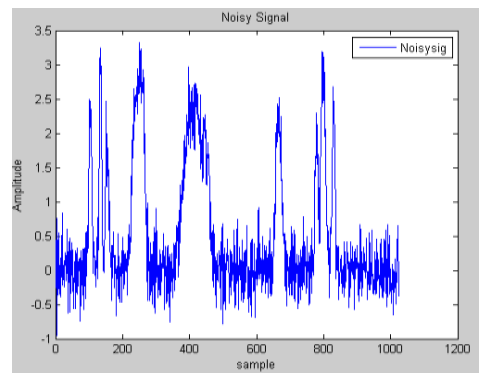

(b)

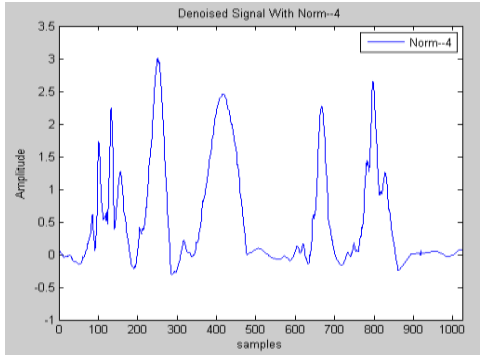

(e)

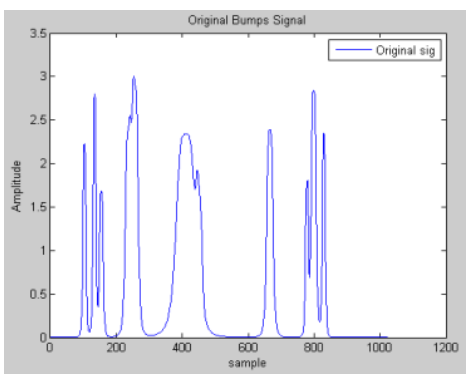

(a)

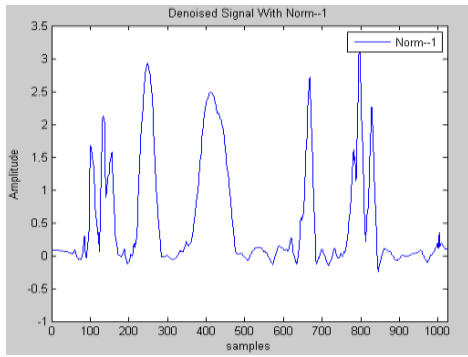

(d)

Figure 4. Results of Applying Different Wavelets to Bumps signal: (a) Pure Bumps Signal, (b) Noisy Signal with Noise Variance $\sigma=0.3$, (c) Denoised Signal by SER Algorithm, (d) Denoised Signal by Norm1 Algorithm, (e) Denoised Signal by DB3standard Wavelets, (f) Denoised Signal by Norm4 Algorithm

In Figure 5, for the 'Heavisine' the "SER" estimated signal looks cleaner and smoother together with better estimation of the sharp feature. The "Norm1" also has a good job but not as clean as "SER". The "Norm4" was able to extract the sharp corner but the smooth regions are not clean enough. The "DB3" has problem for extracting the sharp feature, so the estimated signal is not acceptable.

The 'Doppler' signal may be the most difficult one to denoise out of all the standard signals, especially in the highly oscillating region. In such regions, the wavelet coefficients are not sparse and their amplitudes are similar to those of noise at the fine resolution level. Thus, this is more difficult to extract the original 'Doppler' from the noisy one. As shown in Figure 6, the "SER" method outperforms the other three methods in the sense that the "SER" estimated signals look more like the clean signals than other estimates with acceptable accuracy.

It is standard to evaluate the mean absolute square (MSE) or signal-to-error ratio (SER) of each algorithm for more accurate comparison. The SER is defined as equation (20).

$$
S E R=\frac{\sum_{t=0}^{L-1} x^{2}(t)}{\sum_{t=0}^{L-1}[x(t)-\hat{x}(t)]^{2}}
$$

The SER comparison of applying presented methods to the standard signals are illustrated in Table 2. Each column is related to the specific noise variance. Higher noise variance leads to a lower SER. Different range of noise variance is used to compare the presented method more accurate. These SERs presented in Table 2 confirms the findings mentioned before. The best method in terms of SER is the "SER" method. Two rows are assigned to the "Norm1" and "Norm4" methods because according to Table 1, two different set of scales were selected to design desired wavelets for each method. 


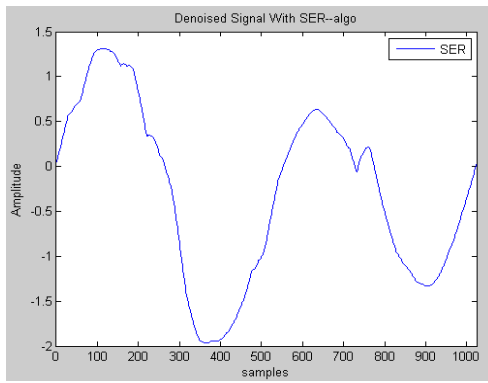

(c)

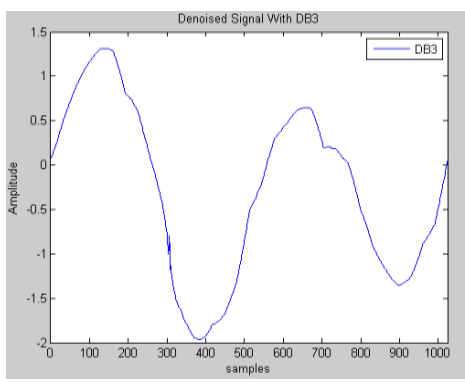

(f)

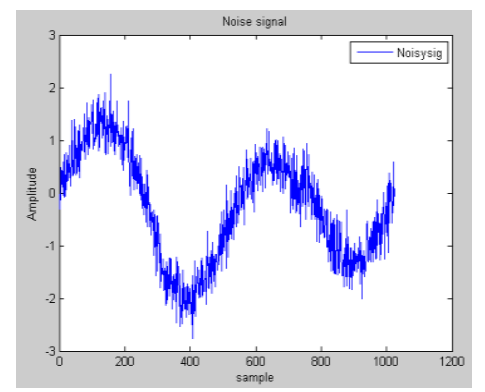

(b)

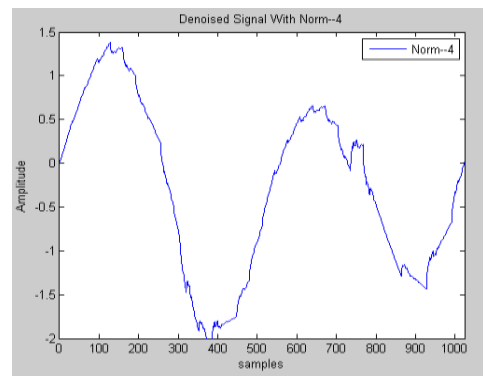

(e)

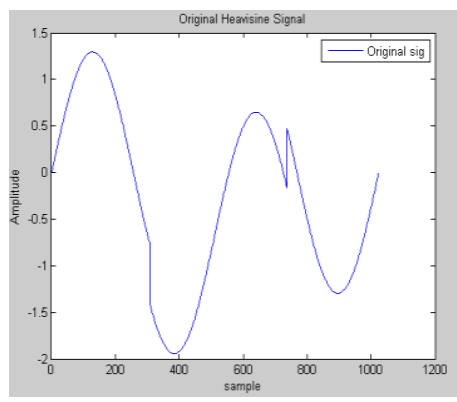

(a)

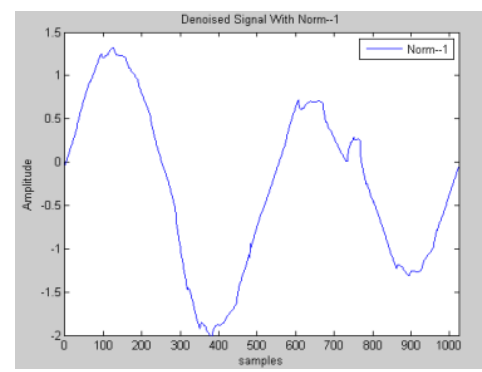

(d)

Figure 5. Results of Applying Different Wavelets to Heavisine Signal: (a) Pure Heavisine Signal, (b) Noisy Signal with Noise Variance $\sigma=0.3$, (c) Denoised Signal by SER Algorithm, (d) Denoised Signal by Norm1 Algorithm, (e) Denoised Signal by DB3standard Wavelets, (f) Denoised Signal by Norm4 Algorithm

Table 2. SER (in dB) Comparison of Different Methods Applied to Standard Signals vs. Changes to The Noise Variance

\begin{tabular}{cccccc}
\hline Signal & Algorithm type & $\sigma=0.1$ & $\sigma=0.2$ & $\sigma=0.5$ & $\sigma=0.8$ \\
\hline Blocks & DB3 & 12.31 & 11.36 & 9.63 & 8.94 \\
& Norm1_algo & 21.9 & 16.79 & 12.22 & 10.75 \\
& Norm4_algo & 14.37 & 13.22 & 11.97 & 11.01 \\
& SER_algo & 19.71 & 16.93 & 12.7 & 10.62 \\
& & & & & \\
Bumps & DB3 & 12.41 & 11.63 & 9.68 & 8.20 \\
& Norm1_algo & 14.95 & 13.33 & 10.64 & 9.26 \\
& Norm4_algo & 14.42 & 12.92 & 10.58 & 9.11 \\
& SER_algo & 14.72 & 13.33 & 11.21 & 9.77 \\
& & & & & \\
Heavisine & DB3 & 28.12 & 24.52 & 19.81 & 16.57 \\
& Norm1_algo & 27.72 & 24.62 & 20.11 & 16.65 \\
& Norm4_algo & 26.11 & 24.11 & 19.83 & 16.42 \\
& SER_algo & 29.23 & 25.54 & 20.33 & 16.92 \\
& & & & & \\
Doppler & DB3 & 21.27 & 17.45 & 12.87 & 10.53 \\
& Norm1_algo & 21.78 & 17.94 & 13.65 & 11.35 \\
& Norm4_algo & 19.83 & 16.85 & 13.06 & 11.23 \\
& SER_algo & 22.13 & 18.31 & 13.78 & 11.45 \\
\hline
\end{tabular}

It should be noticed that noise variance is crucial to the performance of denoising methods. As shown in Table 2, the overall performance of "SER" method is better than others but the "Norm1" method outperforms the "SER" method at Blocks and Bumps signalsfor noise variance less than 0.2. All of the SERs of Table 2 are presented after 100 times repeat and taking average of results achieved, due to random nature of the noise. 


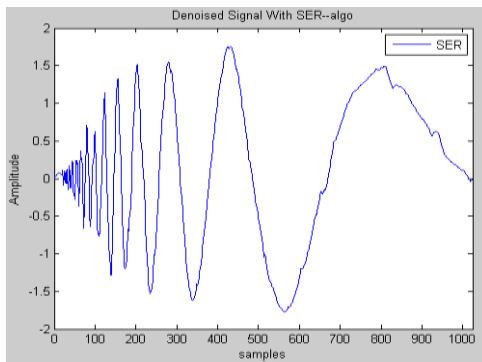

(c)

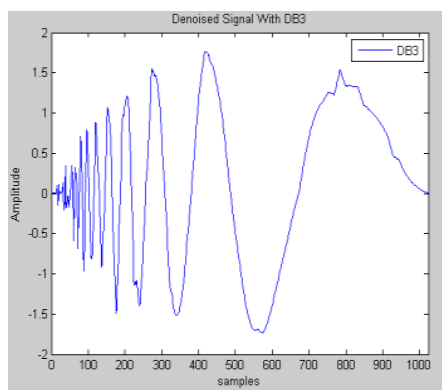

(f)

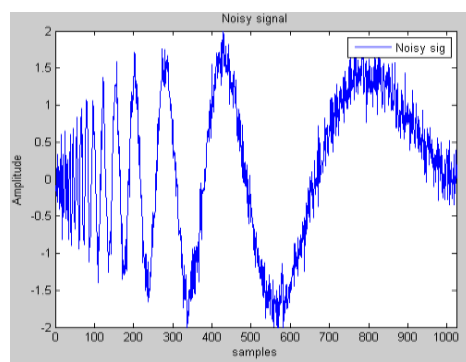

(b)

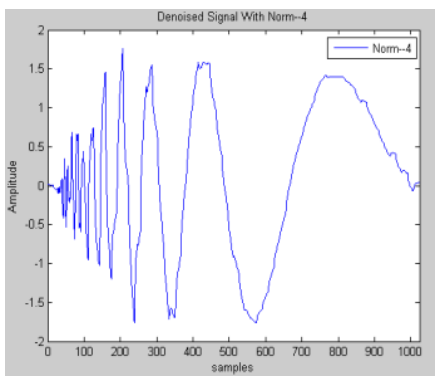

(e)

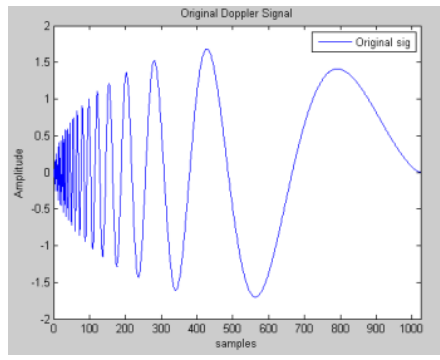

(a)

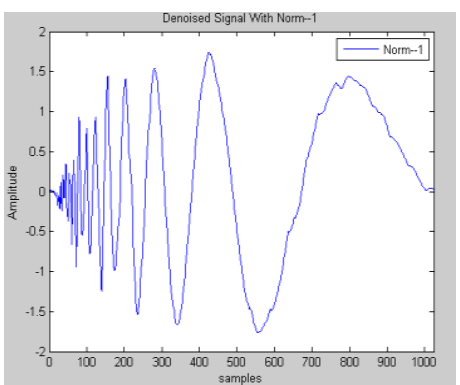

(d)

Figure 6. Results of Applying Different Wavelets to Doppler Signal: (a) Pure Doppler Signal, (b) Noisy Signal with Noise Variance $\sigma=0.3$, (c) Denoised Signal by SER Algorithm, (d) Denoised Signal by Norm1

Algorithm, (e) Denoised Signal by DB3standard Wavelets, (f) Denoised Signal by Norm4 Algorithm

\subsection{Simulated Ultrasound Signal}

In this section, a mathematical model is used to simulate ultrasound signals. This simulated signal could be shown as equation (21) [28]:

$$
x(t)=A \exp \left(-\alpha t^{2}\right) \sin (2 \pi f t+\phi)
$$

where $\alpha=2 \times 10^{12} s^{-2}, \phi=0, A=1$ were used as required parameters. The simulated echo is shown in Figure 7(a). Then, this echo is used as a reference signal for designing wavelets based on the presented criteria. Table 3 illustrates the free parameters $(\theta$ and $\lambda)$ acquired for the proposed algorithms. The denoising results are shown in Figure 7. Simulation is performed by the same steps as described in the previous section.The precise value of the scales depends on parameters such as the signal itself, its coarse level, and features available in it. For signals comprised of low frequency features, we can use smaller scales, whilst for sharper and higher frequency features, a broader scale should be used for optimization purposes. Hence, a scale of 6 to 9 have been used for the simulated echo signal. In all the simulations, signal length is 1024. To have comparable results, all the signals are normalized before simulations in order to have the same power.

Table 3. Free Parameters and the Value of the Cost Function for Simulated Ultrasonic Echo. The Filter Length is $\mathrm{L}=6$

\begin{tabular}{|c|c|c|c|c|c|}
\hline Algorithm type & $\theta_{1}$ & $\theta_{2}$ & $\lambda_{\text {mine }}$ & $\lambda_{d b 3}$ & scale \\
\hline Norm1_algo & 1.19828 & 1.67804 & 6.16372 & 7.5653 & $6,7,8,9$ \\
\hline & 3.52466 & -0.10693 & 0.9792 & 1.4114 & $7,8,9$ \\
\hline Norm4_algo & 3.48622 & 3.01159 & 0.5489 & 1.2572 & $6,7,8,9$ \\
\hline & 3.52352 & 3.03056 & 0.0551 & 0.1446 & $7,8,9$ \\
\hline SER_algo & 1.21436 & 1.69362 & \multicolumn{3}{|c|}{$\sigma_{\text {forwaveletdesign }}=0.3$} \\
\hline
\end{tabular}




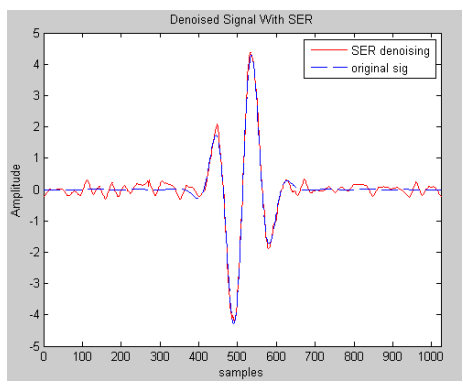

(c)

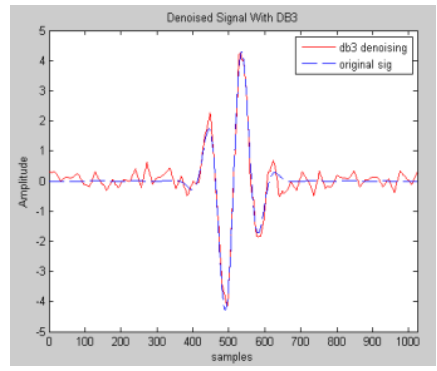

(f)

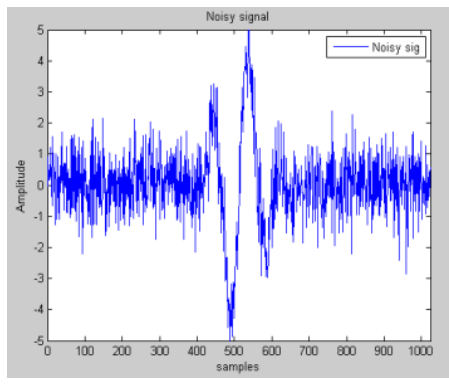

(b)

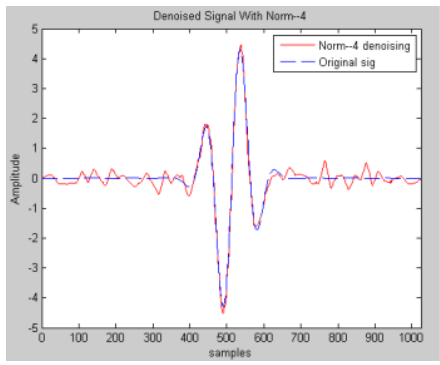

(e)

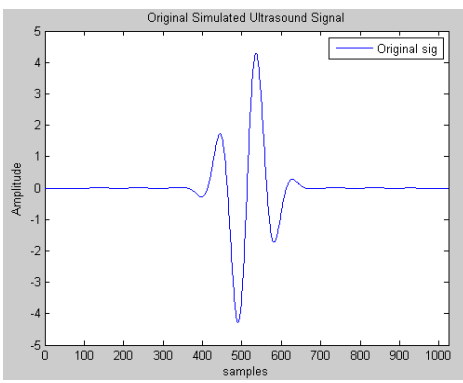

(a)

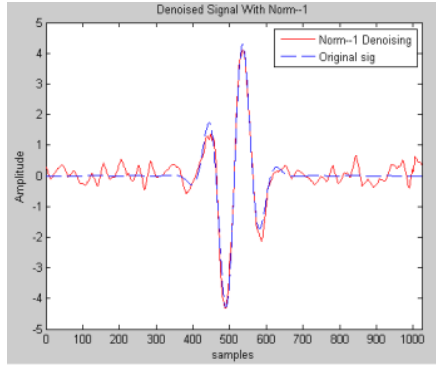

(d)

Figure. 7. Simulated Ultrasound Echo (a) Pure Simulated Signal, (b) Noisy Signal with Noise Variance $\sigma=0.8$, (c) Denoised Signal by SER Algorithm, (d) Denoised Signal by Norm1 Algorithm, (e) Denoised Signal by Norm4 Algorithm, (f) Denoised Signal by DB3standard Wavelets

Estimated signals using presented methods are shown in Figure 7. As seen, the "SER" method outperforms the other three methods in the sense that the "SER" estimated signals is much more similar to the clean one. The "SER" method has a good job at extracting the sharp features and cleaning the flat regions.

The SER comparison of applying presented methods to the Simulated Ultrasonic echo are illustrated in Table 4. Each column is related to the specific noise variance. Here we have different range of noise variance to compare the presented method more accurate. These SERs presented in Table 4 also confirms the findings mentioned before.Comparing these results, the "SER" method results in bigger SER values which confirms its better performance.

Table 4. SER (in dB) Comparison of Different Methods Applied to Simulated Ultrasonic Signal vs. Changes

\begin{tabular}{cccccc}
\multicolumn{8}{c}{ to The Noise Variance $(\sigma)$} \\
\hline Algorithm type & $\sigma=0.05$ & $\sigma=0.1$ & $\sigma=0.2$ & $\sigma=0.5$ & $\sigma=0.8$ \\
DB3 & 31.32 & 27.13 & 22.89 & 17.1 & 13.32 \\
Norm1_algo & 31.55 & 27.74 & 24.15 & 17.93 & 14.02 \\
Norm4_algo & 32.34 & 27.65 & 23.45 & 17.85 & 13.84 \\
& 30.21 & 27.32 & 24.08 & 17.87 & 13.95 \\
SER_algo & 32.62 & 27.81 & 23.52 & 17.64 & $7,8,9$ \\
\end{tabular}

Finally, the performance of the proposed algorithm is compared with other methods in different literatures. The result of this comparison is presented in Table 5. In [29], the undecimated wavelet transform (UWT) was used. Two different UWT processors have been proposed based on two UWT implementation schemes: the "à trous" algorithm and the cycle-spinning scheme. In [30], a new method is presented which is combination of EMD and intrinsic mode function (IMF) techniques. In [31], a comparison of DWT, SWT and WP denoising methods was presented. In [32], a denoising method based on Empirical mode decomposition (EMD) and correlation coefficients analysis is presented. In [33], a new mixed algorithm is presented based on DWT and independent component analysis (ICA) techniques. 
In [34], a fast algorithm is presented to enhance the wavelet denoising parameter. In that paper, an ideal band-pass filter was implemented over common wavelet thresholding process. In [35], the authors presented two sparse deconvolution methods for NDT testing. The first method called "MP" is a special matching pursuit algorithm for deconvolving the noisy data. The second method called "APM" is based on the approximate Prony method. In [36], instead of lower order wavelets, higher order wavelets with large number of coefficients and vanishing moments were used to remove noise from noisy data. In [37], a new threshold processing function is presented which is continuous and higher-order derivative. In [38], different kinds of wavelet base denoising parameters together with thresholding techniques were used to remove the impact of noise on the ultrasonic testing signals of standing trees. The best method among them is selected for comparison.

Table 5. Comparison of Denoising Performance of Various Methods from Different Literatures

\begin{tabular}{ccc}
\hline References & SER results (in dB) & Presented method \\
\hline [29] (2006) & 17.33 & A trous \\
& 20.19 & Cycle spinning \\
[30] (2007) & 20.35 & EMD + IMF \\
[31] (2009) & 18.28 & SWT \\
& 20.23 & WP \\
[32] (2010) & 20.79 & C-EMD based \\
[33] (2010) & 21.83 & WT + ICA \\
[34] (2011) & 21.46 & DWT + BPF \\
[35] (2012) & 22.47 & Omp \\
& 20.09 & apm \\
[36] (2013) & 21.92 & db (36) \\
[37] (2015) & 21.27 & EXP type Threshold function \\
& 22.04 & Ln type Threshold function \\
[38] (2016) & 19.77 & DWT + Thresholding tech \\
& & \\
Proposed methods & 21.91 & Norm 1 \\
& 22.61 & SER \\
\hline
\end{tabular}

Due to the better denoising performance, "SER" and "Norm1" methods were chosen for this comparison. As can be seen, the proposed algorithm "SER" has better denoisingperformance and detection accuracy versus other methods from different literatures.It should be considered that some of these methods have also acceptable denoising performance as "SER" such as [38] and [38].

\section{CONCOLUSION}

In this paper a new method of wavelet design for better noise reduction and feature extraction has been presented in a Gaussian noise model. Two different methods from other literature together with a chosen standard wavelet have been used to compare the accuracy of the propose method.Standard signals such as Doppler, Blocks, Heavisine and Bumps have been used to evaluate the performance of the proposed method. A simulated ultrasonic echo is also used to evaluate performance of the presented methods on the ultrasonic NDT signals. In the standard signals and simulated echo, the "SER" method outperformed the other methods when the signals contained flat and smooth regions and also sharp features such as cups, bumps and corners. Signal to error ratio was used to evaluate the performance of each algorithm. SER Results also have shown that the "SER" method outperformed the other methods due to its larger SER values. A key point of this work could be that, all designed wavelets outperform the standard wavelet in the case of quality and quantity which means, signal-based noised reduction methodsare more effective and accurate. Finally, comparing the proposed algorithm with some other methods from different literatures proved the improved denoising accuracy of the new scheme.

It should be noticed that the performance of these methods are only evaluated on some very specific signal cases, and the current results may not be applicable in all scenarios.Finally, it should be said that there exist more advanced wavelet-based denoising methods which have not been made comparison to in this 
paper. In fact, the proposed algorithm presented in this work can be combined together with each of these advanced methods to become an even more powerful denoising method.

\section{REFERENCES}

[1] J.R. Mohammed, M.S. Shafi, S. Imtiaz, R.I. Ansari, M. Khan, "An efficient adaptive noise cancellation sheme using ALE and NLMS filters", International Journal of Electrical and Computer Engineering (IJECE), vol. 2, no. 3, pp. 325-332, 2012

[2] J.R. Mohammed, "Low complexity adaptive noise canceller for mobile phones based remote health monitoring", International Journal of Electrical and Computer Engineering (IJECE), vol. 4, no. 3, pp. 422-432, 2014.

[3] S. Lakshmikanth, K.R. Natraj, K.R. Rekha, "Noise and vibration reduction in permanent magnet synchronous motors- a review", International Journal of Electrical and Computer Engineering (IJECE), vol. 2, no. 3, pp. 405416, 2012

[4] S. Mallat, "A Wavelet Tour of Signal Processing”. Academic Press, 1998

[5] S. Gopinathan, R. Kokila, P. Thangavel, "Wavelet and FFT based image denoising using non-linear filters", International Journal of Electrical and Computer Engineering (IJECE), vol. 5, no. 5, pp. 1018-1026, 2015.

[6] I. Daubechies, "Orthonormal bases of compactly supported wavelets", Commun. Pure Applied Math., vol. 41, pp. 909-996, Nov. 1988.

[7] A. Cohen, I. Daubechies, J.C. Feauveau, "Biorthogonal bases of compactly supported wavelets", Commun. Pure Applied Math., vol. XLV, pp. 485-560, 1992.

[8] A. Gupta, S.D. Joshi, S. Prasad, "On a new approach for estimating wavelet matched to signal", in Proc. Eighth National Conf. Commun., Bombay, India, Jan. 2002, pp. 180-184. DOI:

[9] A. Gupta, S.D. Joshi, S. Prasad, "A new method of estimating wavelet with desired features from a given signal", $J$. Sig Proc., vol. 85, no. 1, pp. 147-161, January 2005. DOI: 10.1016/j.sigpro.2004.09.008

[10] A. Gupta, S.D. Joshi, S. Prasad, "A new approach for estimation of statistically matched wavelet", IEEE Trans. Signal Process, vol. 53, no. 5, pp. 1775-1793, April 2005. DOI: 10.1109/TSP.2005.845470

[11] A. Tewfik, D. Sinha, P. Jorgensen, "On the optimal choice of a wavelet for signal representation", IEEE Trans. Inf. Theory, vol. 38, no. 2, pp. 747-766, Mar. 1992. DOI: 10.1109/18.119734

[12] P. Gopinath, P. Odegard, P. Burrus, "Optimal wavelet representation of signals and wavelet sampling theorem", IEEE Trans. Circuits Syst. II, Analog Digit. Signal Process. vol. 41, no. 4, pp. 262-277, Apr. 1994. DOI: $10.1109 / 82.285705$

[13] P. Mallat, P. Zhang, "Matching pursuits with time-frequency dictionaries", IEEE Trans. Signal Process., vol. 41, no. 12 , pp. 3397-3415, Dec. 1993. DOI: $10.1109 / 78.258082$

[14] P. Krim, P. Tucker, P. Mallat, and P. Donoho, "On de-noising and best signal representation", IEEE Trans. Inf. Theory, vol. 45, no. 7, pp. 2225-2238, Nov. 1999. DOI: 10.1109/18.796365

[15] J.O. Chapa, R.M. Rao, "Algorithm for designing wavelets to match a specified signal", IEEE Trans. Signal Process., vol. 48, no. 12, pp. 3395-3406, Dec. 2000. DOI: 10.1109/78.887001

[16] W.S. Lu, A. Antoniou, "Design of signal-adapted biorthogonal filter banks", IEEE Trans. Circuits Syst. I, Funam. Theory Appl., vol. 48, no. 1, pp. 90-102, Jan. 2001. DOI: 10.1109/81.903191

[17] M.K. Tsatsanis and G.B. Giannakis, "Principal component filter banks for optimal multiresolution analysis", IEEE Trans. Signal Process. vol. 43, no. 8, pp. 1766-1777, Aug. 1995. DOI: 10.1109/78.403336

[18] C.S. Burrus, R.A. Gopinath, H. Guo, Introduction to Wavelets and Wavelet Transforms. Prentice Hall, 1998.

[19] H. Zou, A.H. Tewfik, "Parameterization of compactly supported orthonormal wavelets", IEEE Trans. Signal Processing, vol. 41, no. 3, pp. 1428-1431, March 1993. DOI: 10.1109/78.205749

[20] B. Molavi, A. Sadr, "Optimum Wavelet Design for Noise Reduction and Feature Extraction", International Symposium on Communications and Information TechnologiesISCIT '07, Sydney, NSW, pp. 1096-1101, Oct. 2007. DOI: 10.1109/ISCIT.2007.4392180

[21] J.L. San Emeterio, E. Pardo, A. Ramos, M.A. Rodriguez, "Ultrasonic grain noise reduction using wavelet processing. An analysis of threshold selection rules", ECNDT 2006 - Poster 138.

[22] D.L. Donoho, I.M. Johnstone, G. Kerkyacharian, D. Picard, “Wavelet Shrinkage: Asymptotia?”, J. Royal Statistical Soc., Series B, vol. 57, no. 2, pp. 301-369, 1995.

[23] D.L. Donoho, I.M. Johnstone, "Adapting to unknown smoothness via wavelet shrinkage", J. Amer. Statistical Assoc. vol. 90, pp. 1200-1224, 1995. DOI: 10.1080/01621459.1995.10476626

[24] D.L. Donoho, I.M. Johnstone, "Ideal spatial adaptation via wavelet shrinkage", Biometrika vol. 81, pp. 425-455, 1994. DOI: $10.1093 /$ biomet/81.3.425

[25] J.M.H. Karel, R.L.M. Peeters, R.L. Westra, K.M.S. Moermans, S.A.P. Haddad, W.A. Serdijn, "Optimal discrete wavelet design for cardiac signal processing", In $27^{\text {th }}$ annual conference on Engineering in Medicine and Biology, Shanghai, China, pp. 2769-2772, 2005.

[26] Zh. Luo, Q. Xiao, "Studey on adaptive wavelet de-noising for measurement signals and its application", In $6^{\text {th }}$ International Conference on Intelligent Systems Design and Applications ISDA '06, pp. 789 -793, 2006. DOI: 10.1109/ISDA.2006.253713

[27] http://www stat.stanford.edu/wavelab. 
[28] R. Demirli and J. Sanaiie. "Model-based estimation of ultrasonic echoes part ii: Nondestructive evaluation applications", IEEE Trans. Ultrason., Ferroelect., Freq. Contr., vol. 48, no. 3, pp. 803-811, 2001. DOI: $10.1109 / 58.920714$

[29] E. Pardo, J.L. San Emeterio, M.A. Rodriguez, A. Ramos, "Noise reduction in ultrasonic NDT using undecimated wavelet transform", J. Ultras, vol. 44, pp. 1063-1067, 2006. DOI: 10.1016/j.ultras.2006.05.101

[30] Y. Mao and P. Que, "Noise suppression and flaw detection of ultrasonic signals via empirical mode decomposition", Russian Journal of Nondestructive Testing, vol. 43, no. 3, pp. 196-203, 2007.

[31] V. Matz, R. Smid, S. Starman, M. Kreidl, "Signal-to-noise ratio enhancement based on wavelet filtering in ultrasonic testing”, J. Ultras, vol. 49, pp. 752-759, 2009. DOI: 10.1016/j.ultras.2009.05.010

[32] M. Sun, Y. Shen, W. Zhang, "A wavelet threshold denoising method for ultrasonic signal based on EMD and correlation coefficients analysis", In $3^{\text {rd }}$ International Congress on Image and Signal Processing (CISP2010), pp. 3992-3996, 2010.

[33] Z. Talebhaghighi, F. Bazzazi, A. Sadr, "Design and simulation of ultrasonic denoising algorithm using wavelet transform and ICA", In $2^{\text {nd }}$ International Conference on Computer and Automation Engineering (ICCAE 2010), 2628 February, Singapore, pp. 739-743, 2010. DOI: 10.1109/ICCAE.2010.5451260

[34] M. Kubinyi and R. Smid, "Ultrasonic denoising with a modified wavelet filter", In the $6^{\text {th }}$ IEEE International Conference on Intelligent Data Acquisition and Advanced Computing Systems: Technology and Applications, 1517 September, Prague, Czech Republic, pp. 479-482, 2011.

[35] F. Bobman, G. Plonka, Th. Peter, O. Nemitz, T. Schmitte, "Sparse Deconvolution Methods for Ultrasonic NDT", $J$. Nondestruct. Eval.vol. 31, pp. 225-244, 2012. DOI: 10.1007/s10921-012-0138-8

[36] A. Praveen, K. Vijayarekha, S.T. Abraham, B. Venkatraman, "Signal quality enhancement using higher order wavelets for ultrasonic TOFD signals from austenitic stainless steel welds", J. Ultras, vol. 53, pp. 1288-1292, 2013. DOI: 10.1016/j.ultras.2013.03.013

[37] Zh. Lanyong, C. An, D. Yixuan, "Research on a novel denoising method of ultrasonic measurement signal", In 3rd International Conference on Mechatronics, Robotics and Automation (ICMRA 2015), pp. 607-612, 2015.

[38] W. Lin, H. Xu, J. Wu, "Study on the denoising processing of ultrasonic testing signal for standing trees based on wavelet transform”, International Journal of Signal Processing, Image Processing and Pattern Recognition, vol. 9, no. 1, pp. 125-134, 2016. DOI: 10.14257/ijsip.2016.9.1.12

\section{BIOGRAPHY OF AUTHOR}

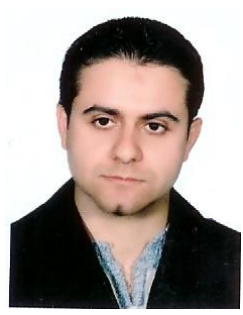

Mohammad Hossein Doost Mohammadi

E-mail: doost.mohammadi@hut.ac.ir

M.H. Doost Mohammadi obtained his M.Sc. degree from the Faculty of Electrical Engineering, Iran University of Science and Technology. Since 2009, he has been Lecturer in Electrical Engineering. Since 2011, he is a Lecturer with the Centers of Electrical and Biomedical Engineering, Hamedan University of Technology, Iran. His interests are mainly Digital Signal Processing and Digital Image Processing and their Implementation systems, especially FPGA. 